\title{
A supervised term ranking model for diversity enhanced biomedical information retrieval
}

Bo Xu $\mathrm{u}^{1,3^{*}}$, Hongfei Lin ${ }^{1 *}$, Liang Yang ${ }^{1}$, Kan Xu1 ${ }^{1}$, Yijia Zhang ${ }^{1}$, Dongyu Zhang ${ }^{1}$, Zhihao Yang ${ }^{1}$, Jian Wang ${ }^{1}$, Yuan Lin ${ }^{2 *}$ and Fuliang Yin ${ }^{1}$

From IEEE International Conference on Bioinformatics and Biomedicine 2018

Madrid, Spain. 3-6 December 2018

\section{Abstract}

Background: The number of biomedical research articles have increased exponentially with the advancement of biomedicine in recent years. These articles have thus brought a great difficulty in obtaining the needed information of researchers. Information retrieval technologies seek to tackle the problem. However, information needs cannot be completely satisfied by directly introducing the existing information retrieval techniques. Therefore, biomedical information retrieval not only focuses on the relevance of search results, but also aims to promote the completeness of the results, which is referred as the diversity-oriented retrieval.
\end{abstract}

Results: We address the diversity-oriented biomedical retrieval task using a supervised term ranking model. The model is learned through a supervised query expansion process for term refinement. Based on the model, the most relevant and diversified terms are selected to enrich the original query. The expanded query is then fed into a second retrieval to improve the relevance and diversity of search results. To this end, we propose three diversity-oriented optimization strategies in our model, including the diversified term labeling strategy, the biomedical resource-based term features and a diversity-oriented group sampling learning method. Experimental results on TREC Genomics collections demonstrate the effectiveness of the proposed model in improving the relevance and the diversity of search results.

Conclusions: The proposed three strategies jointly contribute to the improvement of biomedical retrieval performance. Our model yields more relevant and diversified results than the state-of-the-art baseline models. Moreover, our method provides a general framework for improving biomedical retrieval performance, and can be used as the basis for future work.

Keywords: Biomedical information retrieval, Supervised query expansion, Term ranking model, Diversity-oriented retrieval, Machine learning, Learning to rank

\footnotetext{
*Correspondence: xubo@dlut.edu.cn; hflin@dlut.edu.cn; zhlin@dlut.edu.cn

'Faculty of Electronic Information and Electrical Engineering, Dalian University

of Technology, Linggong Road, Dalian, People's Republic of China

${ }^{3}$ State Key Laboratory of Cognitive Intelligence,iFLYTEK, Hefei, People's

Republic of China

Full list of author information is available at the end of the article
}

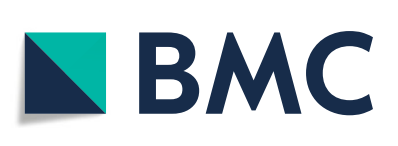

() The Author(s). 2019 Open Access This article is distributed under the terms of the Creative Commons Attribution 4.0 International License (http://creativecommons.org/licenses/by/4.0/), which permits unrestricted use, distribution, and reproduction in any medium, provided you give appropriate credit to the original author(s) and the source, provide a link to the Creative Commons license, and indicate if changes were made. The Creative Commons Public Domain Dedication waiver (http://creativecommons.org/publicdomain/zero/1.0/) applies to the data made available in this article, unless otherwise stated. 


\section{Background}

Biomedical research has developed rapidly in recent years, which leads to a dramatic increase in the number of biomedical research articles. The huge amount of articles make it more and more difficult for researchers to obtain the needed information. Information retrieval (IR) technologies have therefore been introduced to fulfill the information needs. Given a certain query, biomedical retrieval systems aim to provide users with a ranking list of relevant articles. However, the information needs can hardly be fully satisfied due to the ambiguity and uncertainty of queries. This is because the submitted user queries always contain biomedical terminologies in different forms. Namely, a certain terminology may be associated with different synonyms, acronyms and polysemies, which increases the difficulty of term matching in biomedical IR systems. As a result, existing IR methods cannot easily achieve desired performance when directly being applied to solve the problem. Retrieval performance can be enhanced by accurately matching the query terms and potentially relevant terms from articles.

To tackle the problem, biomedical IR systems aim to retrieve the most relevant articles to the given queries, and meanwhile address the diversity of search results for completely meeting the information needs $[1,2]$. The relevance of articles measures the similarity between a given query and the retrieved results. The diversity of the search results is characterized by the covered queryrelated aspects in the outputted ranking list. Each aspect for a given query can satisfy the information need from a certain perspective. All the aspects of the query capture the complete information needs. Therefore, the ratio of covered aspects is used to measure the diversity of search results in biomedical information retrieval. The goal of biomedical IR is thus transformed to retrieve relevant documents covering as many aspects of the query as possible. An aspect of a query can be described with certain biomedical terms, and all the aspects of a query constitute a complete interpretation of the information needs. For example, the biomedical query 'How does P53 affect apoptosis?' involves query-related aspects, such as 'apoptosis regulatory proteins', 'tumor suppressor protein P53' and 'gene expression'. To completely answer the biomedical query, biomedical IR aims to retrieve the most relevant and diversified documents covering these aspects.

Existing studies have been carried out to improve the relevance and the diversity of biomedical search results [3-9]. However, few studies have addressed the diversityoriented biomedical IR using query expansion methods. Query expansion is an effective technique used in IR tasks, which enriches user queries by adding useful terms for interpreting the information needs. General query expansion methods are divided into two categories: unsupervised query expansion (UQE) and supervised query expansion (SQE). UQE methods have been studied for years and have been successfully applied to solve biomedical IR tasks, which measure the importance of expansion terms based on a certain predefined scoring function. The effectiveness of UQE methods varies a lot when using different scoring functions. In fact, different scoring functions measure the usefulness of candidate expansion terms from different perspectives, thus achieving diverse performance.

To completely capture the useful expansion terms, SQE methods have been proposed recently, and effectively improved the quality of the expanded queries [10-13]. SQE methods integrate different UQE methods as term features for learning a supervised model. The model is trained based on a classifier or a ranker for further term refinement. Recent studies have indicated that SQE methods are advantageous over UQE methods in terms of two respects. On one hand, SQE methods count the term importance from different perspectives, which can be considered as the combination of various UQE methods for term refinement. On the other hand, SQE methods can better deal with the retrieval tasks under certain constraints. Since biomedical retrieval focuses on the diversity constraints of search results, we believe that SQE methods can be well adapted to enhance the performance of biomedical IR using effective optimization strategies.

In this paper, we propose a novel supervised term ranking model based on supervised query expansion for diversity-oriented biomedical information retrieval. Our model seeks to obtain the most relevant and diversified search results in biomedical IR. Three optimization strategies are integrated in the proposed model. A biomedical term labeling strategy is designed to capture the relevance and the diversity degrees of the candidate expansion terms.Both the context-based and resourcebased term features are extracted to reflect the usefulness of different terms. A group sampling method is proposed to capture the diversity during the model training process. We modify the loss function of ranking support vector machines using the group sampling method with a diversity-oriented weighting function to improve the quality of selected expansion terms. We evaluate the proposed model on the collections from the TREC Genomics tracks. Experimental results show that our model is effective in improving the performance of biomedical IR.

We summarize the main contributions of this work as follows.

(1) We introduce supervised query expansion for diversity-oriented biomedical information retrieval, and propose a novel method to improve retrieval performance.

(2) We propose to annotate the usefulness of expansion terms by considering the relevance and the diversity 
simultaneously, and extract abundant features for term representations.

(3) We integrate the group sampling and diversityoriented weighting function into the loss function of ranking support vector machines to improve the quality of expansion terms.

\section{Related work}

Query expansion aims to enrich user queries to comprehensively fulfill user information needs in information retrieval (IR), which have been widely used in different IR tasks. Expansion term selection is one of the challenging research topics in query expansion. The quality of selected terms largely affects the accuracy of query expansion. How to select effective terms has attracted much attention in related studies. For example, Lee et al. [14] captured the underlying term associations using abundant term features based on linguistics and statistics. Cao et al. [10] classified the candidate expansion terms based on support vector machines to distinguish good terms from bad terms for expansion. Furthermore, learning-torank methods have been investigated to refine expansion terms $[13,15]$. These supervised machine learning methods for query expansion have been proved to be effective in improving retrieval performance by considering multiple term features, called supervised query expansion. In this study, we mainly focus on term refinement based on modified supervised query expansion.

Existing studies in biomedical information retrieval have integrated query expansion to improve retrieval performance. For example, Srinivasan [16] evaluated the effectiveness of query expansion on MEDLINE collections using the SMART retrieval system. Xu et al. [17] compared query expansion techniques involving local analysis, global analysis and ontology for biomedical literature retrieval. Matos et al. [18] developed a document retrieval and prioritization tool using concept-oriented query expansion to obtain documents with respect to related concepts. Rivas et al. [19] investigated query-specific terms, corpus-specific terms and language-specific terms for biomedical query expansion. These studies demonstrated that query expansion can enhance biomedical IR by considering domain-specific characteristics.

Furthermore, other studies have focused on latent concept expansion in medical and clinical IR tasks, which have also been addressed in general IR tasks. These studies indicated that latent expansion concepts can positively affect biomedical retrieval performance. For example, Bendersky et al. [20] assigned weights on candidate concepts using a weighted dependence model for improving retrieval effectiveness. Zhu et al. [21] identified patient cohorts using mixtures of relevance models to weight query expansion terms for clinical search. TREC 2011 and 2012 medical records tracks have also addressed concept-based retrieval for vertical domain retrieval [22]. In addition, related studies have employed biomedical semantic resources, particularly the $\mathrm{MeSH}$ thesaurus, in query expansion. For example, Oh et al. [23] incorporated the structure of external collections to optimize pseudo relevance feedback. Mao et al. [24] integrated a MeSHenhanced concept layer into a language modeling framework to capture concept associations. Jalali et al. [25] matched concept pairs between queries and documents using a semantic query expansion method. These studies motivate us to optimize query expansion in consideration of domain knowledge.

Inspired by the related work, we propose a supervised query expansion method for diversity-oriented biomedical information retrieval. The proposed method modify our learn-to-rank based query expansion framework [26] from two respects. One is assigning diversity-oriented term labels and extract different types of resource-based term features. The other is developing a novel learning method based on group sampling and diversity weighting function.

\section{Methods \\ General framework}

In this section, we introduce more details about our supervised query expansion framework for diversityoriented biomedical information retrieval. The pipeline of our framework is illustrated in Table 1. Our framework includes two stages: the training stage and the testing stage. In the training stage, we adopt pseudo relevance feedback (PRF) to obtain a large set of candidate expansion terms for each training query. We then represent each candidate expansion term as a feature vector with a ground truth label. In these vectors of terms, we extract both context-based features and resource-based features, and label terms in consideration of their relevance and diversity. We adopt supervised ranking methods to refine the terms by modifying loss function for diversity. In the

Table 1 Supervised query expansion for biomedical information retrieval

\footnotetext{
Algorithm 1 supervised query expansion pipeline

Training the SQE model $M$

1: For each training query $q$, select $k$ candidate terms via PRF

2: Label each term based on the diversity-oriented strategy

3: Represent each term as a feature vector using different term features

4: Train term ranking model $M$ using the modified loss function

Testing the model $M$ in query expansion retrieval

1: For each testing query $q$, select $k$ candidate terms via PRF

2: Represent each term as a feature vector using the term features

3: Apply $M$ to obtain the top $m$ terms for query expansion
} 
testing stage, we apply the learned model to select the expansion terms for query expansion.

We introduce the details of our proposed method in the following sections, including candidate term extraction, term labeling strategy, term features and learning model construction.

\section{Candidate term extraction}

For each query, a set of candidate expansion terms are extracted for further refinement. The candidate expansion terms should be highly correlated with the given query in terms of both relevance and diversity. We adopt a modified pseudo relevance feedback method [26] to extract the terms. The method has been proved to be effective in biomedical information retrieval, which considers term distribution in feedback documents and term distribution in Medical Subject Headings (MeSH) to extract useful expansion terms for further refinement.

\section{Term labeling strategy}

The term labeling strategy is designed to assign ground truth labels on the candidate expansion terms. The labels are treated as the learning targets, and used to compute the ranking loss during model training. A well-defined labeling strategy is crucial for learning a well-performed term ranking model. Existing labeling strategies of supervised query expansion are mostly based on term relevance to a given query. The term relevance can be measured based on its influence on retrieval performance [10]. Specifically, we first conduct an initial retrieval with the original query $q$, and record the retrieval performance as $\operatorname{Eval}(q)$ by any evaluation metric Eval, such as mean average precision. We then conduct another retrieval with both $q$ and a given candidate term $t$, and record its performance as $\operatorname{Eval}(q, t)$. The relevance label for $t$ is determined by comparing $\operatorname{Eval}(q)$ with $\operatorname{Eval}(t, q)$. This method can be formalized as follows.

$$
\operatorname{label}(t)=\left\{\begin{array}{l}
0 \quad \operatorname{Eval}(t, q) \leq \operatorname{Eval}(q) \\
1 \quad \operatorname{Eval}(t, q)>\operatorname{Eval}(q)
\end{array}\right.
$$

Since the diversity is an important factor for biomedical IR, relevance-based labeling may be insufficient for measuring the usefulness of terms. Therefore, we consider the diversity degrees of terms in generating ground truth labels. Query diversity can be reflected by query-related aspects. Aspects of a given query are explicitly described using domain-specific terms in biomedical IR. Therefore, we can measure the diversity of terms based on their occurrences in query-related aspects.

An intuitive way is to generate the diversity-oriented term labels by considering whether a certain term is contained in any query-related aspect. If the term is contained in any query-related aspect, we believe the term is useful and assign the label 1 to the term. Otherwise, we assign the label 0 to the term. Although this labeling strategy seems simple and feasible, it may ignore some potentially useful information of terms: a term contained in several aspects tends to be more diversified than other terms contained in only one aspect. Besides, the influence of terms on retrieval performance is still an important factor in term labeling. Based on the above consideration, we present a new labeling strategy that integrates the diversity and the relevance of terms, which is formulated in Table 2.

In the table, $\operatorname{div}(t)$ is the number of the query-related aspects containing the term $t$. The aspects of queries have been manually annotated in advance on benchmark collections, such as TREC Genomics Tracks, for the diversity-oriented biomedical retrieval task. More aspects containing $t$ indicate that the term can cover more aspects of the query to diversify the search results. Term labels are divided into four types: definitely useful (label 3), partly useful (label 2), probably useful (label 1) and not useful (label 0). The strategy with multiple labels can more accurately measure the ranking loss than that with binary labels, thus producing more effective term ranking models.

\section{Term features}

Terms are represented as feature vectors for learning supervised term ranking models. Each term feature corresponds to one term statistic, reflecting the term usefulness for the given query. We extract two types of term features in our method: the context-based features and the resource-based features. We introduce the definitions of these two feature sets below.

\section{Context-based features}

Context-based features consider the distribution of terms within the retrieval collection. Since textual statistics are always used to measure the term distribution, we adopt different textual statistics as term features. Two types of features are extracted in our framework: features based on term frequency and inverse document frequency (tfidf) and features based on co-occurrences (cooc). These features have been proved effective in our previous work [13].

For the $t f i d f$ based features, term frequency $(t f)$, inverse document frequency (idf) and their combination are treated as different features. Term frequency measures the number of occurrences of certain terms in a document. Inverse document frequency measures the number

Table 2 Diversity-oriented term labeling strategy

\begin{tabular}{llll}
\hline Relevance/Diversity & $\operatorname{div}(t)=0$ & $\operatorname{div}(t)=1$ & $\operatorname{div}(t)>1$ \\
\hline $\operatorname{Eval}(q, t)>\operatorname{Eval}(q)$ & 1 & 2 & 3 \\
$\operatorname{Eval}(q, t) \leq \operatorname{Eval}(q)$ & 0 & 1 & 2
\end{tabular}


of documents with a certain term. These two textual statistics can be combined as tfidf to jointly measure term importance within the entire retrieval collection. We extract these $t f i d f$ based features both within the entire collection and the top-ranked feedback documents from initial retrieval as different term features.

For the cooc based features, term co-occurrences with query terms are considered. Intuitively, if a certain term co-occurs frequently with query terms, the term is more likely to be treated as an expansion term for query enrichment. Therefore, we extract the cooc features based on the number of co-occurrences. We not only limit the scope of co-occurrences in the document level, but also use sliding windows to extract fine-grained term features.

We extract these context-based features to measure term importance and relevance based on term distribution in the retrieval collection. Furthermore, we extract some resource-based features to capture the domainspecific characteristics of biomedical candidate expansion terms.

\section{Resource-based features}

There exist a large amount of semantic resources for biomedical text mining. These resources contain abundant semantic and syntactic information of biomedical terminologies. The resource-based information can be used for modeling the relationship among domainspecific terms. Therefore, we propose to extract domainspecific term features based on biomedical resources. Two widely used resources, Medical Subject Headings (MeSH) and MetaMap, are investigated in our work.

$\mathrm{MeSH}$ has been widely used to index and catalog biomedical articles on biomedical search engines, such as PubMed. The terminologies in MeSH are organized in a tree-based hierarchical manner. The term distribution in $\mathrm{MeSH}$ can reflect term importance in biomedicine. To capture the term information from $\mathrm{MeSH}$, we define two indicators in analogy to the term frequency and inverse document frequency used in IR tasks. We name these two indicators as MeSH-based term frequency $\left(t f_{\mathrm{MeSH}}\right)$ and $\mathrm{MeSH}$-based concept frequency $\left(i d f_{\mathrm{MeSH}}\right.$ ), respectively. $\mathrm{MeSH}$-based term frequency accumulates the occurrences of certain terms in $\mathrm{MeSH}$. If a certain term occurs frequently in $\mathrm{MeSH}$, it may be important in the biomedical domain, and contains much domain-specific information. We define the MeSH-based term frequency as follows.

$$
t f_{\text {MeSH }}\left(t_{j}\right)=\frac{\log \left(\text { freq }\left(t_{j}, M e S H\right)+1.0\right)}{\log |T|}
$$

where $|T|$ represents the number of terms in MeSH. freq $\left(t_{j}, M e S H\right)$ counts the number of occurrences of $t_{j}$ in $\mathrm{MeSH}$.

MeSH-based concept frequency accumulates the number of unique biomedical concepts containing a certain term in $\mathrm{MeSH}$. If more concepts contains a certain term, the term is more likely to reflect domain-specific characteristics. Specifically, this indicator is defined as follows.

$$
i d f_{\text {MeSH }}\left(t_{j}\right)=\frac{M-m\left(t_{j}\right)+1.0}{m\left(t_{j}\right)+1.0}
$$

where $M$ is the number of concepts, and $m\left(t_{j}\right)$ is the number of unique concepts containing $t_{j}$ in MeSH. idf $f_{\mathrm{MeSH}}\left(t_{j}\right)$ measures the importance of $t_{j}$ in $\mathrm{MeSH}$.

Furthermore, inspired by the statistic tfidf used in IR, we combine $t f_{M e S H}\left(t_{j}\right)$ and $i d f_{M e S H}\left(t_{j}\right)$ as a new term feature. The feature is defined as follows.

$$
t f i d f_{M e S H}\left(t_{j}\right)=i d f_{M e S H}\left(t_{j}\right) \log \left(t f_{M e S H}\left(t_{j}\right)+1.0\right)
$$

Besides, we adopt MetaMap to extract more domainspecific term features. MetaMap is a powerful natural language processing tool in biomedicine. This tool has been widely applied in various biomedical text mining tasks [27]. MetaMap is designed by the National Library of Medicine (NLM) to detect domain-specific concepts from biomedical texts. The concepts are from the Unified Medical Language System (UMLS) metathesaurus. The detected concepts can reflect the domain characteristics of the original texts. We therefore extract term features based on the detected concepts from queries.

Specifically, we first expand the original query with a certain candidate expansion term as an expanded query. we then map the expanded query to a concept query using MetaMAP. The concept query contains the canonical forms of Concept Unique Identifiers (CUIs). Intuitively, if the concept query involves more biomedical concepts, the concept query is likely to convey more domain-specific information. The candidate expansion term may be more effective for query expansion. Hence, we define the number of detected concepts as a term feature. This feature is formalized as follows.

$$
\operatorname{concept}(t)=\operatorname{count}\left(t, Q_{\text {expand }}(t)\right)
$$

where $Q_{\text {expand }}(t)$ is the expanded query with the candidate expansion term $t$. count $\left(t, Q_{e} x p a n d(t)\right)$ accumulates the number of occurrences of $t$ in the concept representations of the expanded query. Since MetaMap returns several candidates for an expanded query, the number of returned candidates may also capture the term importance in biomedicine. We define two term features based on this idea.

$$
\begin{aligned}
& \operatorname{conceptnum}(t)=\operatorname{count}_{C U I}\left(t, Q_{\text {expand }}(t)\right) \\
& \operatorname{candidate}(t)=\frac{\sum_{q \in Q_{\text {expand }}(t)}|R(c)|}{\operatorname{count}_{C U I}\left(t, Q_{\text {expand }}(t)\right)}
\end{aligned}
$$

where conceptnum $(t)$ measures the total number of concepts in the concept query at the query level. $|R(c)|$ is the number of returned candidates for the concept $c$ with 
respect to $Q_{\text {expand }}$. We normalize the feature values by the number of concepts contained in the concept query to make the feature values comparable to each other.

All the candidate expansion terms are represented as feature vectors based on the context-based and resourcebased features. The feature vectors are treated as the inputs for model training. The intermediate models are optimized based on pre-defined ranking loss functions towards the ground truth term labels.

\section{Group enhanced loss function for term ranking}

In this section, we introduce the ranking loss function in our method. Loss function measures the difference between the intermediate predictions and their corresponding targets for model selection at the training time, which can be iteratively reduced until the optimal model is yielded. A well-performed loss function for biomedical term selection should count the difference between predicted term labels and the ground truth labels in consideration of both relevance and diversity degrees of terms. To this end, we introduce a group sampling method based on the group enhanced ranking algorithm [28]. The group enhanced ranking algorithm is an effective learning to rank method based on the divide-and-conquer strategy. Learning to rank methods have been widely used in the field of information retrieval to improve the ranking performance, which construct ranking models using supervised machine learning methods.

To adapt group enhanced ranking to biomedical term refinement, we divide the candidate expansion term sets for each query into smaller groups. Each term group contains one term with higher label and several terms with lower labels. We accumulate the losses produced by all the groups to achieve the total loss of intermediate model, and optimize the model by iteratively reducing the total loss. The loss is reduced by gradient descent, particularly on wrongly ranked groups. Therefore, we believe that the group sampling method can yield the final model that focuses on highly useful terms by ranking effective terms at the top of term ranking list, thus improving the quality of the expanded query. The loss function based on group sampling can be formalized as follows.

$$
\operatorname{loss}(k)=\sum_{i=1}^{m} \sum_{j=1}^{n} L\left(f\left(t_{i}^{j}, \omega\right), y_{i}\right)
$$

where $m$ is the number of queries and $n$ is the number of groups for the $i^{t h}$ query. $f$ is the predictive function and $y$ is the target label. The function $L$ characterizes the similarity between the predicted label $f\left(t_{i}^{j}, \omega\right)$ and the target label $y_{i}$. Different versions of $L$ have been used in different learning to rank methods, such as the exponential function and the logarithmic likelihood function. Based on this function, we accumulate the ranking loss of every group of term samples. Since diversity is a key factor to choose the biomedical expansion terms, we further incorporate a diversity-oriented weights on groups to address the diversity of different groups. We formalize the method as follows.

$$
\operatorname{loss}(k)=\sum_{i=1}^{m} \sum_{j=1}^{n}\left(\gamma\left(t_{i}^{j}\right)\right) L\left(f\left(t_{i}^{j}, \omega\right), y_{i}\right)
$$

where $\gamma$ is the diversity-oriented weighting function. Different types of $\gamma$ functions can be adopted in our framework with the underlying idea that more diversified terms should be chosen as expansion terms. We provide one type of $\gamma$ function as follows.

$$
\begin{array}{r}
\gamma\left(t_{i}^{j}\right)=\frac{1}{Z} \times \frac{\operatorname{NumAsp}\left(t_{i}^{j}\right)+1.0}{\operatorname{Total\operatorname {Count}}\left(t_{i}^{j}\right)+1.0} \\
\text { s.t. } 0<\gamma<1
\end{array}
$$

where NumAsp(t) represents the number of aspects containing the term $t$. TotalCount $(t)$ represents the number of occurrences of term $t$ in all the aspects of the given query. $Z$ is a normalization factor to limit the weights within the range of 0 to 1 . Based on the above defined loss function, the constructed term ranking model pays more attention on relevant and diversified terms for in-depth query understanding and complements.

\section{Group enhanced ranking support vector machines for term ranking}

Our diversity-oriented query expansion framework is flexible and can be implemented using different supervised learning methods. In this study, we adopt the ranking support vector machines (RankSVM) [29] to examine the performance of our model using the loss function in Eq. (9).

RankSVM is a variant of the support vector machine algorithm, which is used to solve certain ranking problems via learning to rank. The original purpose of the algorithm was to improve the performance of an internet search engine. The goal of RankSVM is to learn a linear model to minimize the number of pairs of terms in wrong preference orders. Formally, the objective function of RankSVM is defined as follows.

$$
\begin{aligned}
& \min \frac{1}{2} \omega^{T} \omega+C \sum_{i=1}^{m} \sum_{j=1}^{n} \sum_{u, v, y_{u, v}^{i}} \xi_{u, v}^{i, j} \\
& \text { s.t. } \omega^{T}\left(t_{u}^{i, j}-t_{v}^{i, j}\right) \geq 1-\xi_{u, v}^{i, j}, t_{u}^{i, j} \succ t_{v}^{i, j}, \xi_{u, v}^{i, j} \geq 0
\end{aligned}
$$

where $t_{u}^{i, j} \succ t_{v}^{i, j}$ implies that the term $u$ should be ranked ahead of the term $v$ in the $j^{\text {th }}$ group for the $i^{t h}$ query. $C$ is the trade-off coefficient between the ranking loss and the model complexity. 
To apply the group enhanced ranking to RankSVM, we incorporate the diversity-oriented weighting function into the objective function. The modified objective function seeks to compute the ranking loss in consideration of the diversity and relevance of terms. The final form of the objective function is defined as follows.

$$
\begin{aligned}
& \min \frac{1}{2} \omega^{T} \omega+C \sum_{i=1}^{m} \sum_{j=1}^{n} \sum_{u, v, y_{u, v}^{i}} \xi_{u, v}^{i, j} \\
& \text { s.t. } \gamma\left(t_{u}^{i, j}\right) \omega^{T} t_{u}^{i, j} \geq \gamma\left(t_{v}^{i, j}\right) \omega^{T} t_{v}^{i, j}+1 \\
& \quad-\xi_{u, v}^{i, j}, t_{u}^{i, j} \succ t_{v}^{i, j}, \xi_{u, v}^{i, j} \geq 0
\end{aligned}
$$

where $\gamma$ is the diversity-oriented weighting function based on group sampling. This modified objective function is used to compute the ranking loss as the function $L$ in Eqs. (8) and (9) does for model construction. We believe that the learned ranking model based on the modified objective function can select more relevant and diversified terms for biomedical query expansion. The wellconstructed expanded queries will contribute to enhancing the retrieval performance.

\section{Results}

In this section, we evaluate the proposed model with sufficient experiments. We first introduce the experimental settings, and then evaluate the performance in terms of labeling strategy, term feature and loss functions, respectively. We finally report the overall retrieval performance of our framework and provide analysis and discussions on the results.

\section{Experimental settings}

We evaluated the proposed framework on the retrieval collections from 2006 and 2007 TREC Genomics tracks $[1,2]$. The collections are public available and contain 162,259 articles from 49 biomedical journals. The objective for these tracks is to design effective information retrieval systems, which can search for relevant articles and passages given biomedical queries. There are totally 26 queries for 2006 track and 36 queries for 2007 track.

We adopted four evaluation measures, Document MAP, Passage MAP, Passage2 MAP and Aspect MAP. These measures were designed for the tracks as variations of Mean Average Precision (MAP). MAP is a classic evaluation measure used in general IR tasks. The former three measures were designed to evaluate retrieval performance in terms of document-level relevance and passage-level relevance. Aspect MAP aimed to evaluate the retrieval performance in terms of the diversity of the search results.

We implemented the proposed method within the Indri search engine framework [30]. Indri, as a general IR framework, has been widely used in different IR tasks. We indexed articles from the experimental collections with stemmed words and stopword removed in advance. We tuned the parameters of our method for 2006 collection with 2007 queries, and tuned the parameters for 2007 collection with 2006 queries. The selected parameters are reported in Table 3, which have been used in our previous work [26].

To build ranking models based on RankSVM, we performed five-fold cross validations. The reported performance is the average performance on all the five folds. We divided the training set, testing set and validation set by query numbers at the ratio of $3: 1: 1$. The training set was used for model training, the testing set was used for prediction, and the validation set was used for ranking parameter selection. The division follows the standard partition for the learning-to-rank datasets in LETOR [31]. Next, we evaluate the labeling strategy, term feature and loss functions, respectively, and report the overall retrieval performance and discussions.

\section{Performance of labeling strategies}

We conducted the first experiment to examine the effectiveness of the proposed labeling strategy compared to two baseline strategies: One is based on the relevance of terms using Eq. (1), denoted as BinaryRel, and the other is based on whether a term is contained in query-related aspects, denoted as BinaryDiv. We report the retrieval performance in Table 4.

The table shows that the binary relevance strategy achieved relatively good performance in terms of the relevance-based measure Document MAP, while the binary diversity strategy achieved good performance in terms of the diversity-based measure Aspect MAP. This indicates that term labels can guide the model learning process and improve retrieval performance from different respects. Furthermore, the proposed strategy outperformed the other two strategies in terms of both Document MAP and Aspect MAP. This observance indicates that the proposed labeling strategy considering both the relevance and diversity of terms are effective for improving the retrieval performance. One explanation for this finding is that more relevant and diversified terms can be achieved for expansion when the model is optimized towards relevance and diversity of term labels.

\section{Performance of term features}

We conducted the second experiment to examine the effectiveness of different sets of term features, including the context-based set (Context), the resource-based set

Table 3 Parameter settings in our method

\begin{tabular}{llll}
\hline Para. & \#Feedback docs. & \#Expa. terms & \#Cand. terms \\
\hline 2006 & 60 & 30 & 150 \\
2007 & 10 & 30 & 150 \\
\hline
\end{tabular}


Table 4 Effectiveness of different labeling strategies

\begin{tabular}{lllll}
\hline & Document & Passage & Passage2 & Aspect \\
\hline 2006 queries & & & & \\
BinaryRel & 0.3129 & 0.0221 & 0.0271 & 0.2579 \\
BinaryDiv & 0.3095 & 0.0237 & 0.0337 & 0.2635 \\
The Proposed & 0.3282 & 0.0249 & 0.0345 & 0.2828 \\
2007 queries & & & & \\
BinaryRel & 0.3091 & 0.0796 & 0.1093 & 0.2552 \\
BinaryDiv & 0.3252 & 0.0845 & 0.1153 & 0.2721 \\
The Proposed & 0.3337 & 0.0847 & 0.1155 & 0.2723 \\
\hline
\end{tabular}

(Resource) and the combination set with all the features (All). We report the experimental results in Table 5 on the two collections.

From the table, we observe that for 2006 queries, the context-based features outperformed the resource-based features in terms of Document MAP, but less performed in terms of Aspect MAP. The model using all the features achieved the best performance by all the evaluation measures. For 2007 query, the model with the resourcebased features outperformed that based on the contextbased features, and the model using all the features was the most effective in improving the performance. These results implies that both the context-based features and the resource-based features can contribute to the overall performance of the term ranking model. These two feature sets are complementary to each other and jointly improve the performance by combining them together.

\section{Performance of loss functions}

We conducted the third experiment to examine the effectiveness of the proposed loss function in comparison with other loss functions, and report retrieval performance in Table 6. In the table, original ranking loss represents that the model was trained based on the original form of the loss function of RankSVM, group sampling loss represents that the model was trained based on the loss function of RankSVM with group sampling, and weighted group loss represents the proposed form of loss function in Eq. (12).

Table 5 Effectiveness of different feature sets

\begin{tabular}{lllll}
\hline & Document & Passage & Passage2 & Aspect \\
\hline 2006 queries & & & & \\
Context & 0.3168 & 0.0317 & 0.0288 & 0.2613 \\
Resource & 0.3130 & 0.0238 & 0.0335 & 0.2713 \\
All & 0.3282 & 0.0249 & 0.0345 & 0.2828 \\
2007 queries & & & & \\
Context & 0.3037 & 0.0786 & 0.1076 & 0.2428 \\
Resource & 0.3126 & 0.0804 & 0.1096 & 0.2605 \\
All & 0.3337 & 0.0847 & 0.1155 & 0.2713 \\
\hline
\end{tabular}

Table 6 Effectiveness of different ranking loss functions

\begin{tabular}{lllll}
\hline & Document & Passage & Passage2 & Aspect \\
\hline Original loss & 0.3065 & 0.0235 & 0.0335 & 0.2632 \\
Group loss & 0.3088 & 0.0235 & 0.0335 & 0.2641 \\
Proposed loss & 0.3282 & 0.0249 & 0.0345 & 0.2828 \\
2007 queries & & & & \\
Original loss & 0.3226 & 0.0844 & 0.1160 & 0.2667 \\
Group loss & 0.3307 & 0.0841 & 0.1153 & 0.2684 \\
Proposed loss & 0.3337 & 0.0847 & 0.1155 & 0.2713 \\
\hline
\end{tabular}

The table shows that group sampling indeed enhanced the performance of the original RankSVM, and the diversity-oriented term weighting further achieved the best performance. The experimental results indicates that group samples of terms help distinguish the useful terms and useless terms, thus enhancing retrieval performance. The diversity-oriented term weighting further guides the model training for more diversified terms, and improve the diversity of retrieval results in terms of aspect MAP to a large extent.

\section{Overall retrieval performance}

In this section, we report the overall retrieval performance of our model based on the proposed labeling strategy, all the defined features and the weighted loss function with group sampling. We compared our model with state-ofthe-art baseline models.

For the models compared, the query-likelihood language model [32] is a classic retrieval model in IR. The language model is also used as the basic retrieval model in our experiments. Relevance model [33] and term dependency model [15] are two unsupervised query expansion models widely used in different tasks. Support Vector Machine (SVM), RankSVM and ListNet [34] are three learning to rank methods belonging to the pointwise approach, the pairwise approach and the listwise approach, respectively. The SVM-based SQE method has been proved effective in [10]. We report the retrieval performance of all the models in Table 7 on the two collections. Two-tailed paired Student t-tests $(p<0.05)$ were used to examine whether the improvements are significant relative to the baseline models. In the table, an asterisk $(*)$ indicates significant improvements over the RankSVM-based model and a dagger $(\dagger)$ indicates significant improvements over the ListNet-based model.

The table shows that compared to classic retrieval models, unsupervised query expansion methods enhanced the retrieval performance of biomedical retrieval task. Furthermore, the supervised query expansion method improved the performance on both query sets. Our method significantly outperformed other baseline models 
Table 7 Overall retrieval performance of different models for 2006 queries

\begin{tabular}{lllll}
\hline & Document & Passage & Passage2 & Aspect \\
\hline 2006 queries & & & & \\
Language model [32] & 0.3178 & 0.0205 & 0.0239 & 0.1983 \\
Relevance model [33] & 0.3194 & 0.0207 & 0.0240 & 0.2023 \\
Term dependency [15] & 0.3198 & 0.0208 & 0.0254 & 0.1785 \\
SVM-based SQE [10] & 0.3050 & 0.0237 & 0.0292 & 0.2447 \\
ListNet [34] & 0.3216 & 0.0234 & 0.0290 & 0.2256 \\
RankSVM [29] & 0.3065 & 0.0235 & 0.0335 & 0.2632 \\
Our model & $0.3282^{*} \dagger$ & $0.0249^{*} \dagger$ & $0.0345^{*} \dagger$ & $0.2828^{*} \dagger$ \\
2007 queries & & & & \\
Language model [32] & 0.2587 & 0.0646 & 0.0876 & 0.2000 \\
Relevance model [33] & 0.2678 & 0.0720 & 0.0963 & 0.2302 \\
Term dependency [15] & 0.2804 & 0.0683 & 0.0939 & 0.1974 \\
SVM-based SQE [10] & 0.2833 & 0.0729 & 0.0999 & 0.2298 \\
ListNet [34] & 0.2819 & 0.0739 & 0.1012 & 0.2255 \\
RankSVM [29] & 0.3226 & 0.0844 & 0.1160 & 0.2467 \\
Our model & $0.3337^{*} \dagger$ & $0.0847^{*} \dagger$ & $0.1155 \dagger$ & $0.2713^{*} \dagger$ \\
\hline
\end{tabular}

In the table, an asterisk $(*)$ indicates significant improvements over the RankSVM-based model and a dagger $(\dagger)$ indicates significant improvements over the ListNet-based model

in terms of most evaluation measures, which demonstrates the effectiveness of the proposed model. One possible explanation for this finding is that all the modifications of the proposed method based on RankSVM contribute to constructing an effective diversity-oriented term ranking model for choosing high-quality expansion terms to expand the original query, and interpret the query for better fulfilling the information needs in biomedical information retrieval.

To further evaluate our retrieval performance, we compared our results with the median results, the mean results and the best results reported in the 2006 and 2007 tracks of TREC Genomics. The results of the comparisons are presented in Table 8 .

The table shows that the proposed model largely improved the mean and the median results of 2006 official submissions in terms of Document MAP and Aspect MAP, and outperformed the best result of 2007 official submissions in terms of most evaluation measures. The results imply that our method enhanced the relevance and diversity of biomedical retrieval results. We also observe that our method achieved better results in terms of Document MAP and Aspect MAP, but did not perform as well by Passage MAP and Passage2 MAP. This is because these two passage-level measures evaluate the retrieval results at character-level precision, which require extra processing by splitting the retrieved documents into relevant pieces. Our method does not seek to optimize this step, and therefore yields lower performance in terms of these
Table 8 comparison with the best and mean results in the Genomics tracks

\begin{tabular}{lllll}
\hline & Document & Passage & Passage2 & Aspect \\
\hline 2006 queries & & & & \\
Median MAP & 0.3083 & 0.0316 & 0.0345 & 0.1581 \\
Mean MAP & 0.2887 & 0.0347 & 0.0392 & 0.1643 \\
Best MAP & 0.5439 & 0.1012 & 0.1486 & 0.4411 \\
Our model & 0.3282 & 0.0249 & 0.0345 & 0.2828 \\
2007 queries & & & & \\
Median MAP & 0.1897 & 0.0565 & 0.0377 & 0.1311 \\
Mean MAP & 0.1862 & 0.0560 & 0.0398 & 0.1326 \\
Best MAP & 0.3286 & 0.0976 & 0.1148 & 0.2631 \\
Our model & 0.3337 & 0.0847 & 0.1155 & 0.2713 \\
\hline
\end{tabular}

measures. We will optimize our method to enhance these measures in our future work for fine-grained retrieval results.

\section{Discussion}

In this work, we introduce supervised query expansion for term refinement in diversity-oriented biomedical information retrieval. Our model annotates the usefulness of expansion terms by simultaneously considering the relevance and the diversity of terms. The context-based and resource-based features are extracted for comprehensive term representation. In model training, we incorporate the group sampling and diversity-oriented weighting function into the loss function of ranking support vector machines to improve the quality of expansion terms. Overall, we attribute the improvement of the proposed method in biomedical information retrieval in three respects: the term-labeling strategy, the term features and the ranking models. For the term-labeling strategy, we propose to consider the query-related aspects to generate the ground truth labels of candidate terms, which yields more accurate labels during model training. For the term features, we extract both the context-based and the resource-based features, which depict the usefulness of terms more completely from different perspectives and complement each other in constructing the term-ranking models. For the ranking models, we introduce the group sampling and diversity-oriented weights to learn more effective term ranking models. These three aspects jointly contribute to the improvement in retrieval performance, and the proposed framework can also be further optimized in these aspects to enhance biomedical retrieval performance.

\section{Conclusions}

In this study, we propose a novel supervised term ranking model to address the diversity-oriented biomedical 
information retrieval task. Our model is constructed based on the supervised query expansion process. The learned model integrates three novel optimization strategies to select the most relevant and diversified terms for query enrichment. We first propose a diversity-oriented term labeling strategy by considering the diversity degrees of terms. We then represent the candidate expansion terms using both the context-based and resource-based features. To enhance the learned models, we incorporate the group sampling method with a diversity-oriented weighting function into the ranking loss function of RankSVM. Experimental results on TREC collections demonstrate the effectiveness of the proposed model. Our model outperforms the baseline models, and effectively improves the performance of biomedical information retrieval in terms of relevance and diversity. Our model provides a general framework for improving biomedical retrieval performance. Our future work will seek to extract more powerful term features based on other useful biomedical resources, and investigate other effective supervised learning methods for further optimizing the proposed framework.

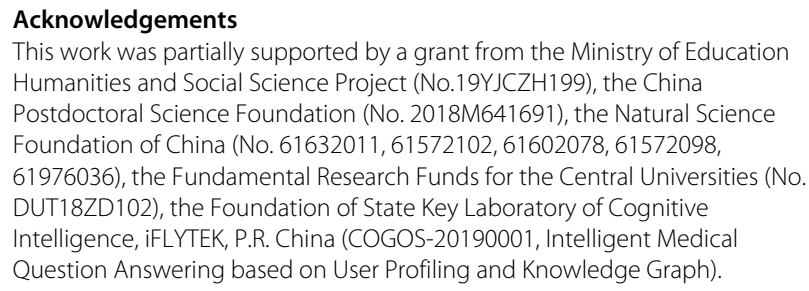

\section{About this supplement}

This article has been published as part of BMC Bioinformatics Volume 20 Supplement 16, 2019: Selected articles from the IEEE BIBM International Conference on Bioinformatics \& Biomedicine (BIBM) 2018: bioinformatics and systems biology. The full contents of the supplement are available online at https:// bmcbioinformatics.biomedcentral.com/articles/supplements/volume-20supplement- 16.

\section{Authors' contributions \\ BX carried out the overall work and experiments. HF contributed to the algorithm design, draft review and modifications. LY contributed to the experiments coding and the draft review. KX contributed to the experiments coding and the draft review. YZ contributed to the experiments coding and the draft review. DZ contributed to the algorithm design. ZY contributed to the algorithm design. JW contributed to the algorithm design. YL contributed to the draft review and modifications. FY contributed to the draft review and modifications. All authors have read and approved of the final manuscript.}

\section{Funding}

Publication costs were funded by a grant from the Ministry of Education Humanities and Social Science Project (No.19YJCZH199), the China Postdoctoral Science Foundation (No. 2018M641691), the Natural Science Foundation of China (No. 61632011, 61572102, 61602078, 61572098, 61976036), the Fundamental Research Funds for the Central Universities (No. DUT18ZD102), the Foundation of State Key Laboratory of Cognitive Intelligence, iFLYTEK, P.R. China (COGOS-20190001, Intelligent Medical Question Answering based on User Profiling and Knowledge Graph).

Availability of data and materials

Not applicable.
Ethics approval and consent to participate

Not applicable.

\section{Consent for publication}

Not applicable.

\section{Competing interests}

The authors declare that they have no competing interests.

\section{Author details}

${ }^{1}$ Faculty of Electronic Information and Electrical Engineering, Dalian University of Technology, Linggong Road, Dalian, People's Republic of China. 'WISE Lab, School of Public Administration and Law, Dalian University of Technology, Linggong Road, Dalian, People's Republic of China. ${ }^{3}$ State Key Laboratory of Cognitive Intelligence,iFLYTEK, Hefei, People's Republic of China.

Published: 2 December 2019

\section{References}

1. Hersh WR, Cohen AM, Roberts PM, Rekapalli HK. Trec 2006 genomics track overview. In: Fifteenth Text Retrieval Conference, Trec 2006. Gaithersburg: TREC; 2006. p. 14-23.

2. Hersh W, Voorhees E. Trec genomics special issue overview. Inf Retrieval. 2009;12(1):1-15.

3. Yin $X$, Huang $X$, Li Z. Promoting ranking diversity for biomedical information retrieval using wikipedia. In: European Conference on Advances in Information Retrieval; 2010. p. 495-507. https://doi.org/10. 1007/978-3-642-12275-0_43.

4. An X, Huang JX. genov: A new metric for measuring novelty and relevancy in biomedical information retrieval. J Assoc Inf Sci Technol. 2017;68(11):2620-35.

5. Karisani P, Qin ZS, Agichtein E. Probabilistic and machine learning-based retrieval approaches for biomedical dataset retrieval. Database. 2018;2018:. https://doi.org/10.1093/database/bax104.

6. Nguyen $\mathrm{GH}$, Tamine $L$, Soulier $L$, Souf N. Learning concept-driven document embeddings for medical information search. In: Conference on Artificial Intelligence in Medicine in Europe; 2017. p. 160-70. https:// doi.org/10.1007/978-3-319-59758-4_17.

7. Cohen T, Roberts K, Gururaj AE, Chen X, Pournejati S, Alter G, Hersh WR, Demnerfushman D, Ohnomachado L, Xu H. A publicly available benchmark for biomedical dataset retrieval: the reference standard for the 2016 biocaddie dataset retrieval challenge. Database J Biol Databases Curation. 2017;2017: https://doi.org/10.1093/database/bax061.

8. Wang Y, Rastegar-Mojarad M, Elayavilli RK, Liu H. Leveraging word embeddings and medical entity extraction for biomedical dataset retrieval using unstructured texts. Database J Biol Databases Curation. 2017;2017:bax091.

9. Wang Y, Liu S, Afzal N, Rastegar-Mojarad M, Wang L, Shen F, Kingsbury P, Liu H. A comparison of word embeddings for the biomedical natural language processing. J Biomed Inf. 2018;87:12-20.

10. Cao G, Nie JY, Gao J, Robertson S. Selecting good expansion terms for pseudo-relevance feedback. In: International ACM SIGIR Conference on Research and Development in Information Retrieval; 2008. p. 243-50. https://doi.org/10.1145/1390334.1390377.

11. LV Y, Zhai CX, Chen W. A boosting approach to improving pseudo-relevance feedback. In: International ACM SIGIR Conference on Research and Development in Information Retrieval; 2011. p. 165-74. https://doi.org/10.1145/2009916.2009942.

12. Zhang Z, Wang $Q$, Si L, Gao J. Learning for efficient supervised query expansion via two-stage feature selection. In: International ACM SIGIR Conference on Research and Development in Information Retrieval; 2016. p. 265-74. https://doi.org/10.1145/2911451.2911539.

13. $X u B$, Lin $H$, Lin $Y$. Assessment of learning to rank methods for query expansion. J Assoc Inf Sci Technol. 2016;67(6):1345-57.

14. Lee CJ, Chen RC, Kao SH, Cheng PJ. A term dependency-based approach for query terms ranking. In: ACM Conference on Information and Knowledge Management; 2009. p. 1267-76. https://doi.org/10.1145/ 1645953.1646114.

15. Lin $Y$, Lin $H$, Jin S, Ye Z. Social annotation in query expansion: a machine learning approach. In: International ACM SIGIR Conference on Research 
and Development in Information Retrieval; 2011. p. 405-14. https://doi. org/10.1145/2009916.2009972.

16. Srinivasan P. Query expansion and medline. Inf Process Manag. 1996;32(4):431-43.

17. Xu X, Zhu W, Zhang X, Hu X. A comparison of local analysis, global analysis and ontology-based query expansion strategies for bio-medical literature search. In: IEEE International Conference on Systems, Man and Cybernetics; 2008. p. 3441-6. https://doi.org/10.1109/icsmc.2006.384651.

18. Matos S, Arrais JP, Maia-Rodrigues J, Oliveira JL. Concept-based query expansion for retrieving gene related publications from medline. Bmc Bioinformatics. 2010;11(1):1-9.

19. Rivas AR Iglesias EL, Borrajo L. Study of query expansion techniques and their application in the biomedical information retrieval. Sci World J,2014,(2014-3-1). 2014;2014(1):132158.

20. Bendersky M, Metzler D, Croft WB. Learning concept importance using a weighted dependence model. In: ACM International Conference on Web Search and Data Mining; 2010. p. 31-40. https://doi.org/10.1145/ 1718487.1718492.

21. Zhu D, Wu ST, Carterette B, Liu H. Using large clinical corpora for query expansion in text-based cohort identification. J Biomed Inf. 2014;49: 275-81.

22. Voorhees EM, Hersh WR. Overview of the trec 2012 medical records track. Washington: TREC; 2012.

23. Oh HS, Jung Y. Cluster-based query expansion using external collections in medical information retrieval. J Biomed Inf. 2015;58(C):70-79.

24. Mao J, Lu K, MuX, Li G. Mining document, concept, and term associations for effective biomedical retrieval: introducing mesh-enhanced retrieval models. Inf Retrieval J. 2015;18(5):413-44.

25. Jalali $V$, Borujerdi MRM. The effect of using domain specific ontologies in query expansion in medical field. In: International Conference on Innovations in Information Technology; 2008. p. 277-81. https://doi.org/ 10.1109/innovations.2008.4781679.

26. $X u B$, Lin $H$, Lin Y. Learning to refine expansion terms for biomedical information retrieval using semantic resources. IEEE/ACM Trans Comput Biol Bioinforma. 2018;PP(99):1-1.

27. Aronson AR. Effective mapping of biomedical text to the umls metathesaurus: the metamap program. Proc AMIA Ann Symp AMIA Symp. 2001;2001(1):17.

28. Lin $Y$, Lin $H$, Ye Z, Jin S, Sun X. Learning to rank with groups. In: ACM International Conference on Information and Knowledge Management. Toronto: ACM; 2010. p. 1589-1592.

29. Cao Y, Xu J, Liu TY, Li H, Huang Y, Hon HW. Adapting ranking svm to document retrieval. In: International Acm Sigir Conference on Research \& Development in Information Retrieval; 2006. p. 186-93. https://doi.org/ 10.1145/1148170.1148205.

30. Strohman T, Metzler D, Turtle H, Croft WB. Indri: A language-model based search engine for complex queries. In: Proceedings of the International Conference on Intelligent Analysis; 2005. http://ciir.cs.umass. edu/pubfiles/ir-416.pdf.

31. Liu T, Xu J, Qin T, Xiong W, Li H. Letor: Benchmark dataset for research on learning to rank for information retrieval. In: International ACM SIGIR Conference on Research and Development in Information Retrieval. Amsterdam: ACM; 2007.

32. Zhai C, Lafferty J. Model-based feedback in the language modeling approach to information retrieval. In: Tenth International Conference on Information and Knowledge Management; 2001. p. 403-10. https://doi. org/10.1145/502585.502654.

33. Croft LV, Bruce W. Relevance based language models. In: International ACM SIGIR Conference on Research and Development in Information Retrieval; 2001. p. 120-7. https://doi.org/10.1145/3130348.3130376

34. Cao Z, Qin T, Liu T, Tsai M, Li H. Learning to rank: from pairwise approach to listwise approach. In: International Conference on Machine Learning; 2007. p. 129-36. https://doi.org/10.1145/1273496.1273513.

\section{Publisher's Note}

Springer Nature remains neutral with regard to jurisdictional claims in published maps and institutional affiliations.

\section{Ready to submit your research? Choose BMC and benefit from:}

- fast, convenient online submission

- thorough peer review by experienced researchers in your field

- rapid publication on acceptance

- support for research data, including large and complex data types

- gold Open Access which fosters wider collaboration and increased citations

- maximum visibility for your research: over $100 \mathrm{M}$ website views per year

At BMC, research is always in progress.

Learn more biomedcentral.com/submissions 